\title{
E. Merck und das Kokain
}

\author{
Zu Sigmund Freuds Kokainstudien \\ und ihren Beziehungen zu der Darmstädter Firma
}

Albrecht Hirschmüller

\section{Summary}

Documents from the archives of the pharmaceutical company, E. Merck, Darmstadt, shed light on research, production, and marketing of cocaine and other coca alkaloids. When cocaine proved to be a local anaesthetic in 1884 the market expanded enormously. The production of E. Merck is compared with that of other companies in Germany and abroad. Freud, who published on cocaine from 1884 to 1887, was in contact with E. Merck and performed clinical studies for them as well as for an American company.

\section{Zusammenfassung}

Anhand der Unterlagen des Firmenarchivs E. Merck in Darmstadt werden Erforschung, Herstellung und Vermarktung des Kokains und seiner Nebenalkaloide beschrieben. Der Siegeszug, den das Kokain als Lokalanästhetikum ab 1884 antrat, spiegelt sich in den Produktionszahlen und Verkaufspreisen. Vergleiche zur in- und ausländischen Konkurrenz werden gezogen. Freud, der 1884-1887 Beiträge über Kokain veröffentlichte, hatte Kontakt zur Firma Merck und führte sowohl in ihrem Auftrag als auch für ein amerikanisches Konkurrenzunternehmen Arzneimittelstudien durch.

\section{Einführung}

Kokain hat heute als Rauschdroge grosse Verbreitung, ist aber als Arzneimittel nicht mehr gebräuchlich. Seine Verordnung unterliegt in Deutschland den strengen Bestimmungen des Betäubungsmittelgesetzes, und selbst für

Priv.-Doz. Dr. med. Albrecht Hirschmüller, Institut für Geschichte der Medizin, Goethestr. 6, D-72076 Tübingen 
Forschungszwecke wäre die Substanz auf legalem Weg nur schwer zu beschaffen.

Im Zusammenhang mit Forschungsarbeiten zu Freuds Kokain-Studien ${ }^{1}$ stellte sich die Frage, wie man sich als forschender oder behandelnder Arzt im Jahr 1884 eigentlich Kokain besorgte und welchen Bedingungen Produktion, Handel und Verwendung der Droge unterlagen.

Freud hat damals bekanntlich das Kokain im Selbstversuch erprobt und seinem Freund Ernst Fleischl, Professor am Physiologischen Institut in Wien, empfohlen, um ihm damit den Entzug vom Morphium zu erleichtern. Es war bekannt, dass Freud über eine Wiener Apotheke Kokain der Darmstädter Firma E. Merck bezog, dass die Substanz sehr teuer war - er musste für ein Gramm ein Zehntel seines Monatsverdienstes ausgeben -, dass er nach anderen Bezugsquellen suchte und im Jahr darauf ein positives Gutachten für ein amerikanisches Konkurrenzpräparat abgab. Wir wissen auch, dass die Firma Merck ihn im Herbst 1884 mit der Untersuchung eines Kokain-Derivats namens Ecgonin beauftragte. Es muss also direkte Kontakte zwischen Freud und Merck gegeben haben.

Eine Anfrage bei der Firma in Darmstadt ergab, dass zwar - vermutlich bei einem Umzug - Unterlagen aus dem 19. Jahrhundert verlorengegangen sind, das Firmenarchiv aber einige wichtige Dokumente zur Geschichte der Erforschung, Herstellung und Vermarktung des Kokains bewahrt hat. Über diese Materialien ist hier zu berichten.

\section{Zur Geschichte der Firma E. Merck}

Seit dem Jahr 1668 besass die Familie Merck in Darmstadt eine Apotheke. ${ }^{2}$ Heinrich Emanuel Merck (1794-1855)3 , Enkel von Johann Heinrich Merck (1741-1791), der mit Goethe in Kontakt gewesen war, übernahm nach seiner

1 Eine Neuedition von Freuds Kokainschriften erscheint demnächst im S. Fischer-Verlag, Frankfurt a.M.

2 Vgl. Geschichte der Merck'schen Engelapotheke zu Darmstadt. Darmstadt 1905; Löw, Carl: Heinrich Emanuel Merck. Mit einer einleitenden Übersicht über seine Vorfahren und die Geschichte der Merck'schen Engel-Apotheke bis zum Anfang des XIX. Jahrhunderts. Hrsg. von E. Merck, Darmstadt. Darmstadt 1951; ders.: Die Chemische Fabrik E. Merck, Darmstadt. Ein Rückblick auf die Geschichte der Firma in Wort und Bild. Aus Anlass des 125jährigen Bestehens. Darmstadt, E. Merck, 1952; ferner insbesondere Possehl, Ingunn: Modern aus Tradition. Geschichte der chemisch-pharmazeutischen Fabrik E. Merck Darmstadt. [Darmstadt] 1989, mit weiterführender Literatur; Dumitriu, Helene: Die wissenschaftliche Entwicklung der Alkaloid-Chemie am Beispiel der Firma Merck in den Jahren 1886-1920. Diss. Pharm. Heidelberg 1993.

3 Heinrich Emanuel Merck führte stets nur seinen zweiten Vornamen, der somit in das Firmensignet «E. Merck» einging. 
Ausbildung im Jahr 1816 die väterliche Apotheke. Er widmete sich insbesondere der Isolierung und Reindarstellung der Alkaloide, denen nach Sertürners Entdeckung des Morphins im Jahr 1804 das besondere Interesse der Zeit galt. 1827 kündigte er in einem «Pharmaceutisch-chemischen Novitäten-Cabinet» an, eine Sammlung von Alkaloiden Ärzten und Apothekern zur Kenntnis zu bringen und dieselben nunmehr «im Grossen darzustellen». Dieses Ereignis wird als Begründung der Firma E. Merck gewertet.

Die Firma nahm einen steten und eindrucksvollen Aufschwung. Sie weitete Firmenprogramm und Umsatz beständig aus, erweiterte ihre Aktivitäten auf die Herstellung von Chemikalien und Stearinkerzen, von Reagenzien und Diagnostika, vergrösserte die Produktionsstätten, verlegte sie schrittweise an die Stadtgrenze von Darmstadt und dehnte die Geschäftsbeziehungen allmählich immer mehr ins In- und Ausland aus. Zwei Söhne Emanuel Mercks, Carl ${ }^{4}$ und Georg ${ }^{5}$, später auch der jüngste Sohn Wilhelm ${ }^{6}$ wurden in eine Geschäftssozietät mit aufgenommen, und in der Folge waren zahlreiche weitere Familienmitglieder nach kaufmännischen, chemischen und pharmazeutischen Ausbildungen gemeinsam in der Leitung von Apotheke und Arzneimittelfirma tätig. ${ }^{\text {? }}$

Kokain produzierte die Firma ab dem Jahr 1862. ${ }^{8}$ Es lassen sich ziemlich präzise Angaben über die seit Beginn der achtziger Jahre produzierten Mengen und die verwendeten Verfahren machen. ${ }^{9}$ Aufschluss geben insbesondere drei verschiedene Manuskripte von Carl Scriba (1854-1929), dem langjährigen Herstellungsleiter der Alkaloid-Abteilung, aus den Jahren 1908, 1919 und $1926 .{ }^{10}$

4 Carl Wilhelm Merck (1823-1885), Eintritt in die Firma 1846.

5 Georg Franz Merck (1825-1873), Eintritt in die Firma 1849.

6 Wilhelm Ludwig Merck (1833-1899), Eintritt in die Firma 1856.

7 Johann Heinrich Emanuel Merck (1951-1904), Kaufmann, Teilhaber bis 1897; Louis Merck (1854-1913), zweiter Sohn von Carl Wilhelm, Eintritt in die Firma 1883; Carl Georg Merck (1856-1936), jüngster Sohn des Carl Wilhelm, Eintritt in die Firma 1884; Emanuel August Merck (1855-1923), ältester Sohn des Georg Franz Merck, Eintritt in die Firma 1883. Willy Ernst Merck (1860-1932), jüngster Sohn des Georg Franz Merck, Eintritt in die Firma 1886; Carl Emanuel Merck (1862-1909), ältester Sohn des Wilhelm Ludwig Merck, Eintritt in die Firma 1889; George Merck (1867-1926), jüngster Sohn von Wilhelm Ludwig, Gründer der Firma Merck \& Co., New York/Rahway, USA. Sämtliche Angaben nach der Stammtafel in Löw (1951).

8 Löw 1952, 1.c. Anm. 2, S. 33; E. Merck, Darmstadt. Bericht über das Jahr 1934. [Darmstadt] 1934, 46. Der Preis betrug damals 16 Mark für eine Drachme (3,6 g). Cocainhydrochlorid taucht in der allgemeinen Preisliste der Firma Merck erstmals 1882 auf (l.c.).

9 Ich danke Frau Dr. Ingunn Possehl und Herrn Winfried Schütt vom Firmenarchiv Merck für die grosszügige Erlaubnis zur Benutzung des Archivs und für vielfältige Hilfe.

10 Scriba, Carl: Die Entwicklung der Fabrik 1 von 1881 bis 1908. Ein Beitrag zur Geschichte des Hauses Merck. Manuskript, den Herrn Dr. Louis Merck, Dr. E. A. Merck, Dr. Willi Merck, Dr. C. E. Merck und Georg Merck aus Anlass der 25-jährigen Jubilaeen der Herrn Dr. Louis Merck und Dr. E. A. Merck in Dankbarkeit gewidmet. [Handschrift, 357 Seiten, Register] 
Tabelle 1. Cocain aus Folia Cocae Peru (1879-1887). Nach Scriba (1919) fol. 90f.; Angaben in $\mathrm{kg}$

\begin{tabular}{lcc}
\hline Jahr & Folia Cocae & Cocain- $\mathrm{HCl}$ \\
\hline $1879-80$ & 25,000 & 0,048 \\
$1880-81$ & 25,000 & 0,052 \\
$1881-82$ & 58,000 & 0,086 \\
$1882-83$ & 137,500 & 0,294 \\
$1883 / 84$ & 654,570 & 1,413 \\
$1884 / 85$ & 8654,900 & 29,919 \\
$1885 / 86$ & 18396,100 & 70,370 \\
$1886 / 87$ & 3629,200 & 12,875 \\
\hline S[umm]a & 31580,270 & 115,012 \\
& & $=0,292 \%$ \\
\hline
\end{tabular}

Scriba gibt in seinem Manuskript von 1908 Zusammenstellungen über die Menge der verarbeiteten Kokablätter und des daraus hergestellten Kokains, die sich auf Unterlagen seiner Vorgänger bzw. Mitarbeiter Freund (bis 1892) und Dürkopf (1907) stützen. 1919 schildert er ausführlich die inzwischen eingetretene Entwicklung, gibt die alten Tabellen nochmals wieder und ergänzt sie durch neue. Bei Unstimmigkeiten bedient er sich auch der Unterlagen der Buchhaltung und bemüht sich, die Angaben aus verschiedenen Quellen vergleichend zu werten. So lassen sich mit Hilfe von Scribas Unterlagen die Entwicklungsstadien der Kokainherstellung gut rekonstruieren.

Bis zum Jahr 1887 bezog die Firma Kokablätter aus Peru und nahm die Extraktion von Kokain in Darmstadt vor. Dann ging man dazu über, von der Firma Kilz \& Co. in Lima ein dort extrahiertes Rohkokain zu kaufen, das man in Darmstadt weiterverarbeitete. Dies spiegeln die Tabellen 1 und 2.

Es ist unschwer zu erkennen, dass Produktion und Verkauf von Kokain aus südamerikanischem Anbau ab der 2. Hälfte des Jahres 1884 einen zunächst explosionsartigen, dann aber stetigen Aufschwung nimmt, der in den Jahren 1902 bis 1906 mit einer Produktion von über 2 Tonnen Kokain jährlich ein Maximum erreicht, nach einem vorübergehenden Rückgang 1907 bis zum Beginn des Ersten Weltkriegs anhält und dann rasch versiegt. Ab 1906 importierte man zusätzlich Kokablätter aus einer Plantage in

1908. FA Merck. Zu Kokain:Fol.26-48, 354-357; ders.: Die Darstellung des Cocains von 1880 bis 1919 [Handschrift = Tätigkeitsbericht Prof. C. Scriba Bd.2, S.3-97 + Zus.]1919. FA Merck. Zu Kokain: S. 3-97 und Zusätze; ders.: Meine Tätigkeit in der chemischen Fabrik von E. Merck, 1. Juni 1881-31. Dezember 1923 [Handschrift, 230 Seiten, Register]. Darmstadt 1926. FA Merck. Zu Kokain: S. 96-102; von diesem Manuskript existiert auch eine mschr. Transkription; die entsprechende Stelle dort S. 56-59. Diese Seiten hat auch Dumitriu, 1.c. Anm. 2, benützt, nicht aber die viel umfangreicheren Manuskripte von 1908 und 1919. Es ist keineswegs so, dass diese «zum Thema nichts Wesentliches, über den genannten Tätigkeitsbericht [1926] hinausgehend, beitragen» (Dumitriu, 1.c., 14). 
Tabelle 2. Cocain. hydrochloric. aus Roh-Peru-Cocain (1886-1916). Nach Scriba (1919) fol. 91; Angaben in $\mathrm{kg}$

\begin{tabular}{|c|c|c|}
\hline Jahr & Roh-Cocain & Cocain- $\mathrm{HCl}$ \\
\hline $1886 / 87$ & 388,715 & 243,495 \\
\hline $1887 / 88$ & 375,284 & 300,224 \\
\hline $1888 / 89$ & 349,832 & 302,831 \\
\hline $1889 / 90$ & 595,208 & 511,120 \\
\hline $1890 / 91$ & 584,588 & 557,467 \\
\hline $1891 / 92$ & 434,037 & 436,548 \\
\hline $1892 / 93$ & 557,451 & 505,173 \\
\hline $1893 / 94$ & 656,304 & 626,105 \\
\hline $1894 / 95$ & 682,853 & 645,928 \\
\hline $1895 / 96$ & 1080,750 & 790,791 \\
\hline $1896 / 97$ & 869,973 & 831,339 \\
\hline $1897 / 98$ & 1818,528 & 1509,053 \\
\hline $1898 / 99$ & 1832,045 & 1552,824 \\
\hline $1899 / 1900$ & 1694,588 & 1564,230 \\
\hline $1900 / 01$ & 1991,314 & 1418,153 \\
\hline $1901 / 02$ & 2115,463 & 1885,734 \\
\hline $1902 / 03$ & 2744,813 & 2453,454 \\
\hline $1903 / 04$ & 2821,085 & 2157,334 \\
\hline $1904 / 05$ & 2884,726 & 2425,599 \\
\hline $1905 / 06^{\mathrm{a}}$ & 2486,751 & 2145,836 \\
\hline $1907^{b}$ & 952,534 & 821,108 \\
\hline 1908 & 1633,666 & 1339,315 \\
\hline 1909 & 1239,450 & 1172,241 \\
\hline 1910 & 3150,631 & 2810,000 \\
\hline 1911 & 2071,450 & 1783,000 \\
\hline 1912 & 1384,025 & 1093,000 \\
\hline 1913 & 1226,345 & 1423,000 \\
\hline 1914 & 790,768 & 533,700 \\
\hline 1915 & & 29,500 \\
\hline 1916 & & 114,500 \\
\hline S[umm]a: & 39413,177 & $\begin{array}{l}34042,602^{c} \\
=86,32 \%\end{array}$ \\
\hline
\end{tabular}

a Zeitraum 1. Juli 1905-31. Dezember 1906; Scriba macht hier die Anmerkung: «2.487 Kilo Roh-Cocain aus Peru + 114 Kilo Roh-Cocain aus New York +508 Kilo Roh-Cocain aus Javablättern + 35 Kilo Roh-Cocain von Knoll».

b Anmerkung Scriba: «853 Kilo Roh-Cocain aus Peru + 82 Kilo Roh-Cocain aus New York + 1.708 Kilo Roh-Cocain aus Javablättern».

c Richtig: 33 982,602; Diff. 60,000.

Java, an der die Firma Merck beteiligt war, und extrahierte in der heimischen Fabrik. Dieser Produktionszweig wurde immer bedeutsamer: Tabelle 3.

Die Produktion von Kokain aus Java-Blättern erreichte, wie man sieht, im Jahr 1913 einen Gipfel mit über 7 Tonnen und betrug auch während des Krieges, als südamerikanisches Material nur noch schwer zu bekommen war, trotz geringerer Ausbeute Mengen von über 1000 kg jährlich.

1906 gründete Merck zusammen mit den Firmen Boehringer in Waldhof bei Mannheim und Knoll in Ludwigshafen eine Interessengemeinschaft, die 
Tabelle 3. Verarbeitete Java-Cocablätter (1906-1918). Nach Scriba (1919) fol. 23 und 92; Angaben in $\mathrm{kg}$

\begin{tabular}{|c|c|c|c|c|}
\hline Jahr & Cocablätter & Roh-Cocain & $\%$ & Cocain- $\mathrm{HCl}$ \\
\hline 1906 & 58967 & 918,9 & 1,558 & 0,323 \\
\hline 1907 & 94018 & 1647,1 & 1,751 & 1060,280 \\
\hline 1908 & 220429 & 3721,3 & 1,688 & 2303,588 \\
\hline 1909 & 238066 & 3971,6 & 1,672 & 3011,114 \\
\hline 1910 & 186127 & 3182,6 & 1,709 & 2431,207 \\
\hline 1911 & 261254 & 4079,9 & 1,561 & 2898,385 \\
\hline 1912 & 422776 & 6552,4 & 1,549 & 4955,548 \\
\hline 1913 & 724189 & 10683,0 & 1,475 & $7259,581^{\mathrm{a}}$ \\
\hline 1914 & 487245 & 7295,3 & 1,497 & 5678,181 \\
\hline 1915 & 203972 & 2965,7 & 1,454 & 234,706 \\
\hline 1916 & 68380 & 829,3 & 1,212 & 332,035 \\
\hline 1917 & - & - & - & 1246,347 \\
\hline \multirow[t]{2}{*}{1918} & 6744 & 71,5 & 1,060 & 1738,846 \\
\hline & $2971867^{b}$ & 45918,6 & & $\begin{array}{l}33472,818 \\
=72,9 \%\end{array}$ \\
\hline
\end{tabular}

a Dumitriu, l.c. Anm. 2, S. 36, nennt etwas abweichende Zahlen ohne genaue Fundstelle.

b Richtige Summe = 2972,167; Diff. 300.

später Präparate unter dem Markensignet MBK auf den Markt brachte. ${ }^{11}$ Es ergab sich auch eine Zusammenarbeit im Bereich der Kokainproduktion. Merck verarbeitete zeitweilig auch ein Rohkokain von Knoll. Während das von Scriba entwickelte Mercksche Benzol-Extraktionsverfahren sich im Anfang der Zusammenarbeit als dem Boehringerschen überlegen erwies, bot nach dem Ersten Weltkrieg das Boehringersche Verfahren der Kokainsynthese aus Rohstoffspaltverfahren wesentliche Vorteile, so dass Merck zu diesem Verfahren überging. ${ }^{12}$

Es wird in der Kokain-Literatur bisher meist angenommen, dass zwar die flüssigen Koka-Extrakte in Alkaloidgehalt und Wirkung unzuverlässig waren, dass aber seit dem von Niemann 1860 erstmals angegebenen Verfahren zur Darstellung von Kokain ${ }^{13}$ der Weg der pharmazeutischen Aufbereitung für immer feststand und vollkommen verlässliche Präparate vorlagen. Scribas Unterlagen machen dagegen deutlich, dass man auch bei angeblich reinen Kokainpräparaten mit erheblichen Unterschieden im Alkaloidgehalt und infolgedessen auch in der physiologischen Wirkung zu rechnen hatte. Denn selbst bei dem hohen Standard, den die Firma Merck an ihre Produkte anlegte, wurden am Herstellungsverfahren immer noch Verbesserungen

11 Vgl. Possehl, l.c. Anm. 2, S. 66, 74.

12 Scriba 1926, l.c. Anm. 10, Transkription S. 57f.

13 Niemann, Albert: Über eine neue organische Base in den Cocablättern. Göttingen, Vandenhoeck \& Ruprecht, 1860. Zugl. Diss. phil. Göttingen 1860. 
Tabelle 4. Gesamtproduktion von Beginn bis 31. Dezember 1918. Nach Scriba (1919) S. 93; Angaben in kg

\begin{tabular}{llccc}
\hline & Blätter Peru & Roh-Cocain & Blätter Java & Cocain-Hcl \\
\hline $\begin{array}{l}\text { aus Perublättern } \\
\begin{array}{l}\text { aus Peru-Roh-Cocain } \\
\text { aus Javablättern }\end{array}\end{array}$ & 31588 & 39413 & & 115,017 \\
\hline S[umm]a: & $31518^{\mathrm{a}}$ & 39413 & 2972172 & 64042,602 \\
\hline
\end{tabular}

a Richtig: 31588.

vorgenommen, und man versuchte, den Reinheitsgrad zu erhöhen und die Kokain-Ausbeute zu optimieren.

Scriba gibt 1919 auch eine Tabelle, in der die gesamte Kokainproduktion der Firma nach den drei Produktionswegen zusammengestellt ist: Tabelle 4.

Insgesamt produzierte die Firma Merck bis 1918 also nicht weniger als 67 Tonnen Kokain, das entspricht mehr als 100 Millionen Einzeldosen zu $50 \mathrm{mg}$, wie Freud sie verwandte. Man weiss nicht, welcher Teil davon wirklich in den Praxen der Augenärzte und Chirurgen verwandt wurde - Kokain wurde nach der Jahrhundertwende als Lokalanästhetikum mehr und mehr von den synthetischen Lokalanästhetika Novocain und Procain abgelöst und welcher Teil in die Hände derjenigen gelangte, die Kokain als Rauschdroge konsumierten. Gesetzliche Bestimmungen zu einer wirksamen Kontrolle des Handels mit Kokain gab es jedenfalls noch längst nicht. Erst die internationalen Bemühungen zur Eindämmung des Rauschdrogenkonsums nach dem Ersten Weltkrieg führten zu einer drastischen Veränderung der Marktsituation. ${ }^{14}$

\section{Freuds Beziehungen zur Firma Merck}

Was wissen wir nun über Freuds Beziehungen zur Firma Merck? Jones schreibt, Freud habe das Präparat bei Merck in Darmstadt bestellt, das Gramm habe zu seiner Bestürzung nicht wie erwartet 33 Kreuzer, sondern 3 Gulden 33 Kreuzer gekostet, und offenbar erhielt er die Substanz Anfang

14 Die Bestimmungen der Haager Opium-Konvention von 1912, die auch das Kokain betrifft, wurden wegen des Krieges erst 1925 gültig. Deutschland hatte im Vorgriff darauf 1920 ein Opiumgesetz erlassen. Vgl. Maier, Hans W[olfgang]: Der Kokainismus. Geschichte / Pathologie. Medizinische und behördliche Bekämpfung. Leipzig, Georg Thieme, 1926, 233-247; Schendzielorz, Petra: Die Anfänge der Betäubungsmittelgesetzgebung in Deutschland. Unter besonderer Berücksichtigung der Opiumstelle Berlin und des Pharmazeuten Otto Anselmino (1873-1955). Diss. pharm. Berlin 1988. 
Mai $1884 .{ }^{15}$ Findet sich dafür ein Beleg im Archiv der Firma Merck? Bedauerlicherweise sind Korrespondenzen aus jenen Jahren nicht erhalten geblieben, so dass Briefe, die zwischen Freud und der Firma gewechselt worden sind, als verloren gelten müssen. Erhalten ist aber ein Geschäftsbuch von 1883-1886, in dem alle jene Bestellungen und Lieferungen verzeichnet sind, die durch Vorauskasse oder Nachnahme bezahlt wurden. So geschah es offenbar vor allem bei kleineren Bestellungen von Medikamenten und Chemikalien. Unter den Kunden finden sich Kliniken und Sanatorien, Ärzte und Apotheker, aber auch Goldschmiede und Photographen sowie Personen ohne Berufsangabe. Das Buch enthält ein sorgfältig geführtes Register, das die Namen der Kunden mit ihren Wohnorten aufführt.

Zwar erscheinen weder Freud noch Fleischl in diesem Register. Es findet sich aber mit Datum vom 24. April 1884 ein Eintrag, aus dem hervorgeht, dass eben die Apotheke, aus der Freud sein Kokain zunächst bezog, ${ }^{16} \mathrm{C}$. Haubner's Engel-Apotheke in Wien, Am Hof 6, per Post «1 Kistel» erhielt, enthaltend 15 gr. Cocain. mur. sowie 5 gr. Cocain. pur., das Gramm zu je 6.50 Mark, fernerhin 50 gr. Acid. gynocardic. ${ }^{17}$ zum Preis von 13.00 Mark, zuzüglich Packung für eine Gesamtsumme von 143.75 Mark, die per Nachnahme bezahlt wurden (vgl. Faksimile 1).

Man darf sicher sein, dass diese Bestellung durch Freuds Interesse induziert war. Offenbar hat der Apotheker grosse Erwartungen in die neue Droge gesetzt, da er nicht nur sogleich eine grössere Menge einkaufte (20 Gramm entsprechend 400 Einzeldosen), sondern sie auch an Freud unter dem eigenen Einkaufspreis abgab: Freud bezahlte 3.30 Gulden; 6.50 Mark entsprechen aber knapp 4 Gulden.

Am 31. Mai bezog das Physiologische Institut, gewiss zu Händen von Ernst Fleischl, direkt 10 Gramm Kokain zu einem Sonderpreis von 5 Mark pro Gramm (vgl. Faksimile 2). Ob man sich in der Firma Merck schon hier eine Kooperation mit dem renommierten Institut Brückes versprach?

Freuds erste Kokain-Studie, in der er die Literatur ausführlich referierte, die Verwendungsmöglichkeiten der Droge zusammenstellte und über seine eigenen Erfahrungen berichtete, erschien im Juli 1884, aber erst Kollers Entdeckung, dass das Kokain als Lokalanästhetikum am Auge zu verwenden

15 Jones, Ernest: Das Leben und Werk von Sigmund Freud. Bd. 1: Die Entwicklung zur Persönlichkeit und die grossen Entdeckungen 1856-1900. Übers. von Katherine Jones. Bern, Stuttgart 1960, 104.

16 Freud, Sigmund [1884e]: Über Coca. In: Zbl. ges. Ther. 2 (1884) 289-314. Hier: 299.

17 Säure aus Gynocardia odorata, einer in Indien heimischen und gegen Lepra sowie als Fiebermittel eingesetzten Pflanze. Vgl. Hagers Handbuch der pharmaceutischen Praxis für Apotheker, Ärzte, Drogisten und Medicinalbeamte. Neu bearb. und hrsg. von B. Fischer und C. Hartwich. Bd. 1. Berlin 1900, 1280. 


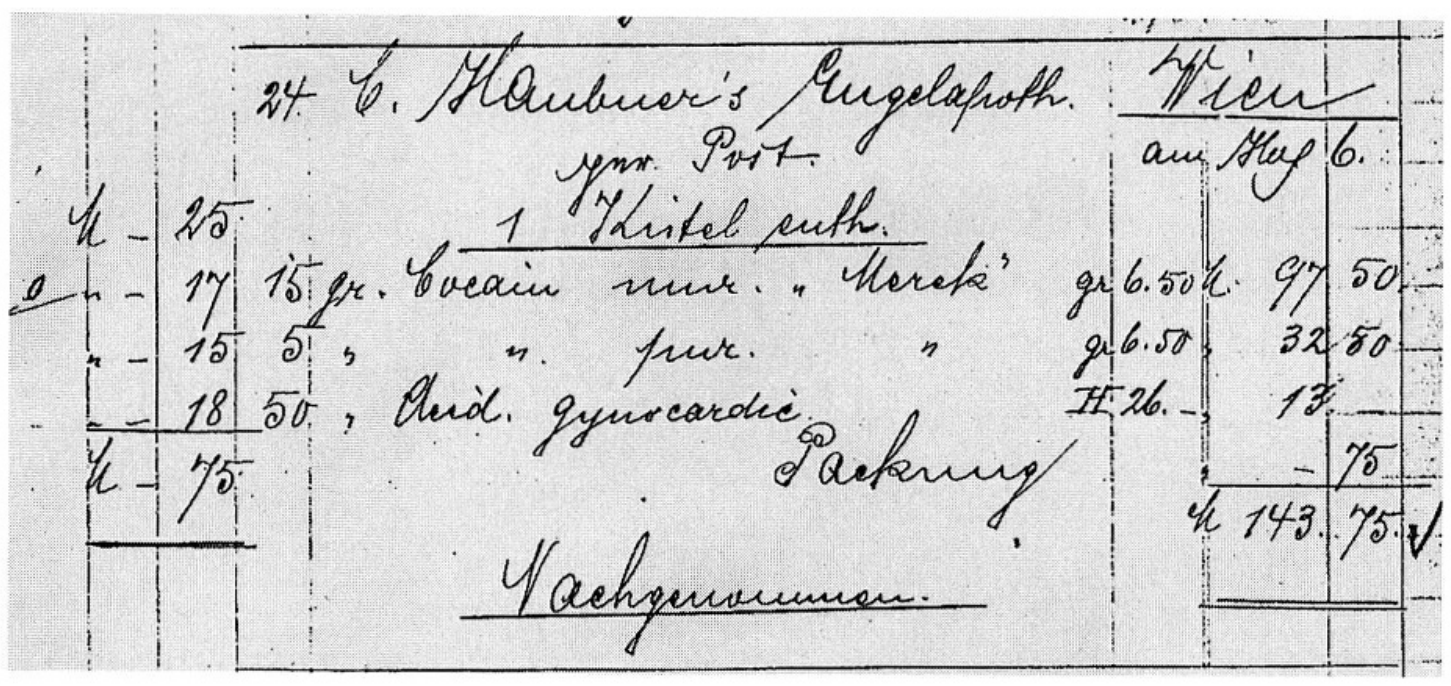

Faksimile 1. Eintrag aus dem Geschäftsbuch 1883-1886 der Firma Merck: Kokain-Bestellung von C. Haubners Engelapotheke in Wien vom 24. 4. 1884.

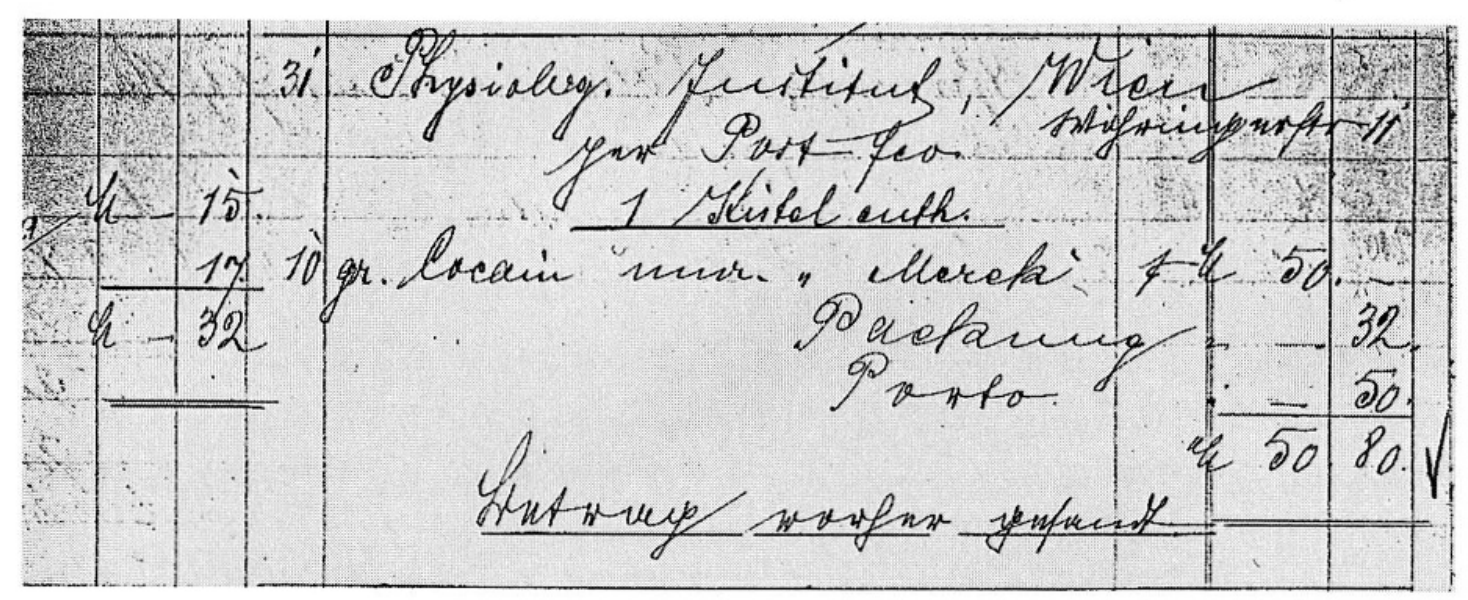

Faksimile 2. Eintrag aus dem Geschäftsbuch 1883-1886 der Firma Merck: Kokain-Bestellung des Physiologischen Instituts in Wien vom 31. 5. 1884.

war, bekanntgemacht auf dem Augenärztekongress in Heidelberg Mitte September und ausführlich in Wien Mitte Oktober, führte zu einer sprunghaften Zunahme des Interesses an der Droge. Addiert man die Kokainbestellungen aus dem Geschäftsbuch, so liegen sie zwischen Januar und Oktober bei monatlich 10-20 Gramm, im November und Dezember etwa bei 60 Gramm und im Jahr 1885 bei mehreren Kilogramm pro Monat.

Die steigende Nachfrage erlaubte eine weitere Anhebung des Preises: Im November und Dezember stieg der Preis von 6 auf 15 Mark, im Februar 1885 erreichte er mit 23 Mark sein Maximum, um dann bei steigendem Angebot und bei wachsender Konkurrenz durch eine amerikanische Firma bis zum 
Oktober 1885 auf 4 Mark und bis zum Jahr 1887 auf 85 Pfennige pro Gramm zurückzugehen. ${ }^{18}$

Wir wissen, dass die Firma Merck spätestens im Herbst 1884 durch Fleischl von Freuds Kokainexperimenten Kenntnis bekam. Der Firma war nämlich Fleischls hoher Kokain-Verbrauch aufgefallen, und sie schrieb ihn deshalb an, wohl um sich zu erkundigen, welche wissenschaftlichen Erkenntnisse man im Wiener Physiologischen Institut über die Substanz gewonnen hatte. ${ }^{19}$ Fleischl hat offenbar geantwortet und über seine und Freuds Erfahrungen berichtet, natürlich ohne darauf hinzuweisen, dass er selbst die Droge als Ersatz für Morphin einnahm. Freud schrieb am 5. Oktober nach Darmstadt und übersandte seine Studie. Bald darauf verschickte die Firma ein ausführliches «Circular» über das Kokain, das umgehend in den Monatsblättern für Augenheilkunde und auch in anderen Zeitschriften abgedruckt wurde, in dem auf Freuds Studie hingewiesen wurde.$^{20}$ Fleischl selbst wird in diesem Text als Gewährsmann genannt, was nur durch die Annahme erklärlich ist, dass er selbst sich gegenüber Merck so dargestellt hat.

\section{Ecgonin}

Erstes Resultat des Kontakts war, dass die Firma Freud anbot, gegen Honorar ein Derivat des Kokains, das Ecgonin, physiologisch zu untersuchen, was Freud gerne akzeptierte, nachdem er sich für die notwendigen Tierversuche - anders als bei Kokain lagen solche für das Ecgonin noch nicht vor - der Mitarbeit Fleischls versichert hatte. Für die Firma Merck bot der gute Name des Wiener Physiologischen Instituts von Ernst Brücke wohl Gewähr für solide Arbeit. Freud bekam $100 \mathrm{~g}$ der Substanz geschickt, und am 21. Oktober 1884 begannen die Experimente. ${ }^{21}$

Die Arbeit gestaltete sich jedoch unerfreulich. Fleischl erwies sich als unzuverlässiger Mitarbeiter, und die Tierversuche zogen sich hin. Freud begann unvorsichtigerweise sogar mit dem Selbstversuch, noch ehe die Toxizität ausreichend festgestellt war. ${ }^{22}$ Die Wirkung war jedoch enttäuschend, sieht man

18 Dies ist den Geschäftsbüchern und den im Merck-Archiv erhaltenen Preislisten im einzelnen zu entnehmen.

19 Brief Freuds an Martha Bernays, 5. 10. 1884, Israëls, Han: Het geval Freud. 1. Scheppingsverhalen. Amsterdam, Uitgeverij Bert Bakker, 1993, 225.

20 Merck, E.: Cocain und seine Salze. [Offene Correspondenz.] In: Klin. Mbl. Augenhk. 22 (1884b) 428-434; die handschriftliche Fassung dieses Rundschreibens ist im Firmenarchiv erhalten geblieben. Der Wortlaut ist mit dem veröffentlichten identisch.

21 An Martha, 11. und 21. 10. 1884, Israëls, l.c., 226.

22 Freud an Martha, 21. 10. 1884, Israëls, l.c. 
davon ab, dass Freud jenen berühmten «scharfen» Traum, von dem er am 3. November berichtete, auf die Einnahme von Ecgonin zurückführte. ${ }^{23}$

Wie kam es, dass eine grosse und berühmte Arzneimittelfirma den Auftrag zur experimentellen und klinischen Prüfung einer neuen Substanz an einen jungen Wiener Krankenhausarzt vergab? Verfügte man nicht über eigene und bessere Möglichkeiten? Tatsächlich hat die Firma Merck in jener Zeit keine eigene pharmakologische Abteilung geführt und auch keinerlei Tierexperimente vorgenommen. Noch für Jahrzehnte musste man sich der Zusammenarbeit mit firmenfremden Wissenschaftlern versichern, so mit dem Erlanger Pharmakologen Heinz, später mit dem Heidelberger Pharmakologen Gottlieb, auf dessen Anregung die Firma 1924 das rechtsdrehende Pseudokokain unter der Bezeichnung Psikain in Verkehr brachte, so auch mit Richard Willstätter, dem 1898 die Strukturaufklärung und 1902 die Totalsynthese des Kokains gelang. ${ }^{24}$

Freud hat eigens für die Prüfung des Ecgonins Überlegungen angestellt, wie sich die erwartete, Körperkraft und psychische Fähigkeiten stimulierende Wirkung des Ecgonins am Menschen objektivieren lassen könnte. Das Ecgonin zeigte zwar keine signifikanten Effekte, aber die neuen experimentellen Methoden liessen eine Objektivierung der Allgemeinwirkung des Kokains selbst zu, und so baute Freud zu einer eigenen Studie aus, was zunächst nur als Vorarbeit für die Ecgoninuntersuchung gedacht gewesen war: Mit einem Dynamometer fand sich eine messbare Steigerung der Körperkraft unter Kokaineinwirkung; als Nebenbefund stiess Freud auf eine gesetzmässige tageszeitliche Schwankung der Körperkraft. Die gleichfalls untersuchte Reaktionszeit erwies sich dagegen nicht als signifikant verkürzt. ${ }^{25}$

Die weitere chemisch-pharmazeutische Erforschung des Kokains und seiner Derivate war für die Firma Merck natürlich von grösstem Interesse. So ist es nicht verwunderlich, dass sich die beiden Familienmitglieder, die zu jener Zeit noch studierten, nämlich Wilhelm Ernst Merck und Carl Emanuel Merck, in ihren Dissertationen mit dem Thema Kokain beschäftigten:

Wilhelm Ernst («Willy») Merck, Enkel des Firmengründers, führte im Frühjahr 1885 im neu eröffneten chemischen Laboratorium von Prof. Ladenburg in Kiel eine Untersuchung über das Kokain durch, mit der er im Jahr

23 Vgl. Freud, Sigmund [1960a]: Briefe 1873-1939. Ausgew. und hrsg. von Ernst und Lucie Freud. 2. erw. Aufl. Frankfurt a.M. 1968, 408.

24 Vgl. Löw 1952, l.c. Anm. 2, S. 58; Hoppe, Brigitte: Aus der Frühzeit der chemischen Konstitutionsforschung: die Tropaalkaloide Atropin und Cocain in Wissenschaft und Wirtschaft. München, Düsseldorf (Deutsches Museum. Abhandlungen und Berichte 47, 1979, Heft 3), bes. $41-43$.

25 Freud, Sigmund [1885a]: Beitrag zur Kenntnis der Cocawirkung. In: Wiener med. Wschr. 35 (1885) Sp. 129-133; vgl.zu Methodik und Ergebnissen Hirschmüller, Albrecht: Dyanometrie im 19. Jahrhundert. 1994 (im Druck). 
1886 promoviert wurde. ${ }^{26} \mathrm{Er}$ schilderte in seiner Dissertation zunächst auf zehn Seiten die traditionelle Kenntnis der Koka-Pflanze und die chemische Darstellung des Kokains. Seine eigene Arbeit schloss an die Forschungen von Niemann und Lossen an. Er widmete sich besonders dem Benzoyl-Ecgonin, einem Nebenprodukt, das bei der Darstellung des Kokains anfiel, und der Resynthese von Kokain aus dieser Substanz. Ferner untersuchte er Kokainhomologe wie Cocäthylin und Cocaisopropylin und fand beide Substanzen in Tierversuchen gleichsinnig, aber bedeutend schwächer wirksam als Kokain. Es gelang ihm aber nicht, die chemische Struktur des Ecgonins schlüssig aufzuklären.

Hieran schloss sich die Arbeit seines Vetters Carl Emanuel Merck an. Hatte er schon bei der Promotion seines Vetters als Opponent fungiert, ${ }^{27}$ so setzte er nun im Winter 1887/88 die chemische Untersuchung des Kokains und Ecgonins fort und beschrieb ein neues Derivat, das er in einer vorläufigen Mitteilung Ecgonidin nannte. Ein Konkurrent namens Einhorn behauptete daraufhin jedoch, denselben Körper schon vor ihm dargestellt zu haben, so dass Merck die Studien beendete, als Dissertation lediglich seine bis dahin gefundenen Ergebnisse veröffentlichte und statt dessen einen zweiten Teil «Über Furfuräthenpyridin» anschloss. ${ }^{28}$ Beide Studien eint der direkte Bezug zu den wirtschaftlichen Interessen des Hauses Merck und der Versuch, durch Optimierung der erwünschten pharmakologischen Wirkung und durch Verringerung der Giftigkeit die Nutzbarkeit von Kokainderivaten zu erhöhen.

\section{Die Konkurrenz}

Auf dem deutschen Markt für Kokain hatte die Firma Merck bis zum Ersten Weltkrieg kaum ernsthafte Konkurrenten. Die Firma Trommsdorff in Erfurt, die ein als zuverlässig geltendes Kokain anbot, wurde 1890 von Merk aufgekauft ${ }^{29}$ mit anderen kleineren Produzenten wie Gehe \& Co. in Dresden, Knoll in Ludwigshafen und Boehringer in Mannheim-Waldhof traf man 1906 Absprachen, die der Firma Merck fast eine Monopolstellung sicherten. ${ }^{30}$

Auch in Frankreich zog man vielfach die Präparate Mercks denen eigener Produzenten vor. ${ }^{31}$ Neben den eigentlichen Kokain-Produkten hatten vor

26 Merck, Willy [Wilhelm Ernst]: Über Cocain. Diss. phil. Kiel 1886.

27 Vgl. l.c., Titelblatt.

28 Merck, Carl Emanuel: Über Furfuräthenpyridin und über Cocain. Diss. phil. Kiel 1888.

29 Possehl, l.c. Anm. 2, S. 56.

30 L.c., 66f.; Scriba 1926, l.c. Anm. 10, S. 58 f.

31 Dumitriu, 1.c. Anm. 2, S. 25 f. 
allem die Koka-Zubereitungen der Firma Mariani weite Verbreitung gefunden. Der in Paris lebende Korse Angelo Mariani hatte sich bereits 1865 einen alkoholischen Koka-Wein als Vin Mariani patentieren lassen. Dieser Wein nahm nach 1884 einen geradezu phantastischen Aufschwung und wurde auch nach Amerika exportiert. ${ }^{32}$ Mariani selbst schrieb wissenschaftliche Bücher, in denen er Koka warm empfahl. ${ }^{33}$ Er rühmte sich zahlloser Schreiben berühmter Zeitgenossen, die seinen Koka-Wein lobten und weiterempfahlen. Seit dem Jahr 1894 gab er Alben heraus, in denen diese Zuschriften abgebildet waren. Begeisterte Zeugnisse kamen von Gelehrten und Künstlern, Politikern und kirchlichen Würdenträgern, Ärzten und Entdeckungsreisenden. Sarah Bernhard, Thomas Edison, Herbert George Wells, Henrik Ibsen, Henri Poincaré, Auguste Rodin, Emile Zola oder auch Papst Leo XIII. finden sich darunter. ${ }^{34}$ Die Zuschriften spiegeln die Erwartungen, die die Zeitgenossen in die Wirkungen der Koka setzten, von der Aufhellung trüber Stimmungen und der Stimulation zu Kreativität und Tatkraft bis zur Stärkung der Singstimme oder zur Steigerung der sexuellen Potenz.

In Amerika begann John Pemberton 1885 in Atlanta, Georgia, einen kokahaltigen Wein nach Marianis Vorbild zu vertreiben. Ein Jahr später entfernte er den Alkoholbestandteil, fügte den Extrakt der coffeinhaltigen Colanuss hinzu und nannte sein Produkt Coca-Cola. Offensichtlich in massiver Unterschätzung der kommerziellen Möglichkeiten verkaufte er wenig später diesen Handelsnamen und die Herstellungsformel, und nach Hinzufügung von Kohlendioxyd fand Coca-Cola weltweit ungeahnten Anklang. Erst die «Pure Food and Drug Act» von 1906 veranlasste die Firma, den Kokainbestandteil aus der Coca-Cola-Formel zu entfernen. ${ }^{35}$

Kehren wir aber aus dem paramedizinischen Markt in den Bereich der eigentlichen Arzneimittel zurück. Hier erwuchs der Firma Merck Mitte der achtziger Jahre in den USA entscheidende Konkurrenz. ${ }^{36}$ Auch in Amerika $32 \mathrm{Vgl}$. Coca erythroxylon (Vin Mariani). Its uses in the treatment of disease. With notes and comments by prominent physicians. 3. Aufl. Paris, Mariani \& Co. [1884]; Kennedy, Joseph: Coca Exotica. The Illustrated Story of Cocaine. Rutherford, Madison, Teaneck und zugl. New York, London, Toronto 1985, 63, Abb. nach S. 65, 83-85.

33 Mariani, [Angelo]: La coca et la cocaïne. Paris 1885; ders.: La coca et ses applications thérapeutiques. Paris 1895.

34 Figures contemporaines tirées de l'Album Mariani. Bd. 1-14. Paris 1894-1925; die kostbar gebundenen Exemplare mit den Originalzuschriften finden sich ebenso in der Pariser Nationalbibliothek wie die in kleiner Auflage gedruckten Faksimile-Ausgaben; eine kleine Auswahl der Blätter geben wieder: Eyguesier, Pierre: Comment Freud devint drogman. Etude sur la coca et la cocaïne à la Belle Epoche. Paris 1983 (Bibliothèque des Analytica); Kennedy, l.c. Anm. 32.

35 Vgl. Kahn, E. J.,jr.:The Big Drink. The story of Coca-Cola.2. Aufl. New York, Random House, 1960; The Coca Leaf and Cocaine Papers. Hrsg. von George Andrews und David Solomon. New York, London, Harcourt Brace Jovanovich, 1975, 10-12.

36 Die Verhältnisse auf dem amerikanischen Arzneimittelmarkt in den Jahren 1884 und 1885 sind aus einer Artikelserie von Eduard Robinson Squibb (1819-1900), Apotheker und Arz- 
waren zunächst vor allem flüssige Kokain-Extrakte von zweifelhafter Qualität angeboten worden. ${ }^{37}$ Gute Koka-Blätter waren teuer und schwer zu bekommen, so dass ein gewissenhafter Hersteller im Juli 1884 schrieb, er schäme sich, nur ein Produkt von minderer Qualität und hohem Preis anbieten zu können und stelle die Produktion ein. ${ }^{38}$ Auch waren die zur Extraktion erforderlichen Lösungsmittel Äther und Alkohol so hoch besteuert, dass ein Import des Merckschen Kokains lange Zeit billiger war als eine eigene Produktion.

Der Marktführer, Parke, Davis \& Co. in Detroit, hatte sich schon länger um eine Verbreitung von Kokapräparaten bemüht und ein fluid extract angeboten. Der Mitinhaber George S. Davis war auch der Verleger der Therapeutic Gazette, jener Detroiter Zeitschrift, in der seit 1878 zahlreiche Mitteilungen über die Substanz und ihre Anwendungen erschienen waren und der auch Freud viele Einzelheiten entnommen hatte.

Bald nach Bekanntwerden von Kollers Entdeckung beauftragte die Firma ihren Chefbotaniker, den Apotheker Henry H. Rusby, in Südamerika Ursprung, Produktion und Verwendungsweise der Koka zu erforschen und Geschäftsbeziehungen anzuknpüfen. ${ }^{39}$ Rusby reiste Anfang 1885 nach La Paz in Bolivien. Es gelang ihm, eine Schiffsladung Kokablätter zu kaufen und auf dem Pazifik nach Norden zu schicken. Monate später aber musste er erfahren, dass die Ladung wegen der bolivanischen Revolution nicht weitertransportiert worden war und noch immer am Isthmus von Panama lag. Rusby sah nun klar, dass der einzig gangbare Weg sein würde, an Ort und Stelle ein Rohkokain zu extrahieren, das dann viel leichter transportiert und daheim verfeinert werden konnte, ein Weg, den dann 1886 auch die Firma Merck be-

neimittelhersteller in New York, recht genau zu rekonstruieren: Squibb, [Edward Robinson] [1884a]: Erythroxylon Coca. In: The pharmceut. j. and transactions 15 (1884-1885) 145-147; ders. [1884b]: Cocaine. In: The pharmaceut. j. and transactions 15 (1884-1885) 465-467; ders. [1885a]: Hydrochlorate of cocaine, or muriate of cocaine. In: Chem. News 51 (1885) 90-92, 98-100,112-114; ders. [1885b]: Hydrochlorate of cocaine, or muriate of cocaine. In: The pharmaceut. j. and transactions 15 (1884-1885) 774-776,796-798; ders. [1885c]: Coca at the source of supply. In: The pharmaceut. j. and transactions 16 (1885-1886) 47-49; ders. [1885d]: Hydrochlorate of cocaine. In: The pharmaceut. j. and transactions 16 (1885-1886) 67-69. Wiederabdruck der gesamten Serie in: Squibb, Edward Robinson: Collected papers. Bd. 1.2. Hrsg. von Klaus Florey. [Princeton, N.J.], The Squibb Institute for Medical Research, 1988, $1217-1274$.

37 Neben dem grössten Hersteller, Parke, Davis \& Co. in Detroit, werden Produkte von F. W. Foucar, E. R. Squibb und von Eimer and Amend, alle in New York, erwähnt; vgl. Knapp, $\mathrm{H}$ [einrich]: Hydrochlorate of cocaine - experiments and application. In: Medical Record 26 (1884) 461-463.

38 Squibb [1884a], l.c. Anm. 36.

39 Rusby, H[enry] H[urd]: Cocoa [!] at Home and Abroad. In: Therapeutic Gazette, Detroit 12 (1888) 158-165. [Gekürzte deutsche Übers. in Springer, Alfred: Kokain. Mythos und Realität. Eine kritisch dokumentierte Anthologie. Wien, München, 1989, 22-24]; Rusby, Henry H[urd]: Jungle memories. New York, London, 1933. 
schritt. In einem Zimmer seines Hotels in La Paz experimentierte Rusby unter improvisierten Bedingungen, und als seine Apparatur Feuer fing, gelang es ihm nur mit knapper Not, sie von der Terrasse zu werfen und damit eine Brandkatastrophe zu verhindern..$^{40}$

Die Firma Parke-Davis widmete sich nun intensiv der Produktion und Vermarktung von Kokainpräparaten. Zunächst verstand man es, den Markt künstlich zu verknappen und den Preis hochzuhalten. ${ }^{41} \mathrm{Ab}$ April 1885 aber stieg das Angebot an guten Koka-Blättern, und die Preise sanken. ${ }^{42}$ Mitte des Jahres 1885 versandte man eine Broschüre, ${ }^{43}$ in der nach einer langen historischen Einleitung auf 26 Seiten die aktuelle Kokainliteratur referiert wurde. Neben dem Reinalkaloid bot die Firma verschiedene Kokain-Salze an, ferner ein Cocaine case mit 5 Kapseln zu je 1 grain Kokainmuriat, einem Fläschchen für Lösungen, einer Pipette, einem Kamelhaarpinsel, einer Spritze für subkutane Injektionen und einer Gebrauchsanweisung zum Preis von \$2.00-3.00. Ende des Jahres kam eine neue, umfangreichere Broschüre heraus, ${ }^{44}$ in der nun alle erdenklichen Verwendungsmöglichkeiten des Kokains genau beschrieben und auch kritische Stimmen nicht ganz verschwiegen wurden. Inzwischen offerierte die Firma auch einen Koka-Wein, KokaZigaretten, ein inhalierbares Produkt und eine besonders wohlschmeckende Zubereitung ohne Adstringentien und Bitterstoffe («Coca Cordial»). ${ }^{45}$

Die Firma Parke-Davis hatte damit zu kämpfen, dass das Mercksche Präparat als bedeutend zuverlässiger galt. Die Firma tat, was auch heute noch in vergleichbaren Situationen versucht wird: Sie beauftragte einen Wissenschaftler, eine vergleichende Untersuchung der beiden Präparate anzufertigen und eine gutachterliche Stellungnahme abzugeben. Wer kam für ein sol-

40 Rusby 1933, l.c., $33 \mathrm{ff}$.

41 Der Preis für Merck-Kokain stieg vom Sommer 1884 bis zum Januar 1885 von \$ 2.50 auf $\$ 13.00$, während das als schlechter geltende amerikanische Kokain jetzt für $\$ 7.70 \mathrm{zu}$ haben war.

42 Squibb nahm die Produktion wieder auf und bot ein eigenes Kokain zu folgenden Preisen an: Preisliste vom 9.2.1885: Hydrochlorate of cocaine 30c/grain $[\bumpeq \$ 4.60 / \mathrm{g}] ; 1.7 .1885: 10 \mathrm{c} /$ grain $(\$ 1.53 / \mathrm{g}) ; 1.7 .1889: \$ 5.25 / \mathrm{oz}[\stackrel{\wedge}{=} \mathrm{c} / \mathrm{grain} \triangleq \$ 0.15 / \mathrm{g}]$. Firmenarchiv Squibb, Princeton, N.J.; ich danke Herrn Dr. Klaus Florey, Princeton, für freundliche Unterstützung.

43 [Parke, Davis \& Co.]: Reports on the application of hydrochlorate of cocaine in ophthalmology, otology, laryngology, gynaecology, genito-urinary, nasal, dental, and general surgery. Compiled from the medical literature. Detroit, New York, Scientific department Parke, Davis \& Co., 1885 (Working bulletins for the collective investigation of new remedies).

44 [Parke, Davis \& Co.]: Coca erythroxylon and its derivatives. A resumé on their history; botanical origin; production and cultivation; chemical composition; therapeutic application; physiological action; and medicinal preparations. Embracing reports on their employment in general and minor surgery; ophthalmology; otology; laryngology; gynaecology; genito-urinary, nasal and dental surgery; in the treatment of the alcohol and opium habits; in general medicine; [...]. Compiled by the scientific department of Parke, Davis \& Co. Detroit, New York, Parke, Davis \& Co., 1885.

45 L.c., 130. 
ches Gutachten in Frage? Das Wort eines Amerikaners hätte wohl als nicht unvoreingenommen gegolten, das Urteil eines Europäers dagegen würde schwerer wiegen. Der Betreffende musste Erfahrung in der Beurteilung der Kokainwirkung haben und durch wissenschaftliche Methodik von möglichster Objektivität ausgewiesen sein. Sie fand einen solchen Gutachter in Sigmund Freud.

Freud übernahm den Auftrag gegen ein Honorar von 60 Gulden im April 1885. ${ }^{46}$ Seine für das Parke-Kokain sprechende Stellungnahme wurde im August in der Wiener Medizinischen Presse gedruckt, ${ }^{47}$ und zwar innerhalb eines Artikels von Gutt, der behauptete, Merck könne nicht liefern und sei zu teuer, und der recht unverhohlen für die Präparate der Firma Parke warb. ${ }^{48}$

Die Firma Merck aber setzte sich zur Wehr. Am 25. Oktober druckte die Wiener Medizinische Presse einen offenen Brief von E. Merck mit folgendem Wortlaut: ${ }^{49}$

Die Nr. 32 der «Wiener Mediz. Presse» laufenden Jahrgangs bringt einen Artikel über Cocain, der in Betreff meiner Firma verschiedene Irrtümer enthält.

Die Arbeit ist unter dem Scheine einer rein wissenschaftlichen Untersuchung lediglich als eine im Interesse einer amerikanischen Firma erfolgte Publikation aufzufassen.

Ohne mich dieser Konkurrenz-Firma gegenüber auf irgend ein Für oder Wider einzulassen, erlaube ich mir in den folgenden Zeilen [die] mein Haus betreffenden, den Thatsachen nicht entsprechenden Fakten des qu. Artikels richtig zu stellen.

Eine Aufgabe der Cocain-Fabrikation ist meinerseits nie erfolgt; ich bin auch zu jener Zeit noch prompt lieferungsfähig geblieben, als die Beschaffung des Rohmaterials mit ernsten Schwierigkeiten zu kämpfen hatte.

Mein Cocain besitzt in seinem jetzigen Zustande keine gelbliche, sondern eine rein weisse Farbe. Bei Aufnahme der Fabrikation konnte man allerdings von einem «Stich in's Gelbliche» reden; doch betone ich, dass, als die Konkurrenz mit der Cocainproduktion begann, mein Präparat bereits auf der jetzigen Höhe technischer Vollkommenheit stand.

Die Bemerkung, dass sich das amerikanische Cocain «erheblich billiger» stelle als mein Fabrikat, ist ebenfalls unrichtig. Der Preis des Alkaloides musste ein hoher sein, solange sich der Werth der Kokablätter auf einer abnormen Höhe bewegte. Seitdem sind Rohmaterial und Cocain billiger geworden.

Wenn ich mein Präparat eine Kleinigkeit über dem Preisniveau der Konkurrenz halte, so glaube ich dazu umso mehr berechtigt zu sein, als der Käufer meiner Marke die Garantie hat, ein völlig brauchbares Cocain zu erhalten, ein Präparat, welches sich in ungezählten Fällen bewährt hat. Für den Arzt eine nicht zu überschätzende Tatsache!

Nach dem Gesagten muss es unverständlich bleiben, wenn der Verfasser des in Rede stehenden Artikels die Aufmerksamkeit «auf Amerika richten» und dem amerikanischen Cocain die «grösste Zukunft» prognostizieren zu sollen glaubt.

Darmstadt, den 1. Oktober 1885.

E. Merck.

46 Jones, l.c. Anm. 15, S. 119.

47 Freud, Sigmund [1885e]: Gutachten über das Parke Cocaïn. In. Wiener med. Presse 26 (1885) Sp. 1036.

48 Gutt: Neue Arzneimittel und Heilmethoden. Über die verschiedenen Cocainpräparate und deren Wirkung. In: Wiener med. Presse 26 (1885) Sp. 1035-1038.

49 Wiener med. Presse 26 (1885) Sp. 1373. 
Es ist unklar, ob Freud den Abdruck seiner Stellungnahme in dem beschriebenen Kontext gebilligt hat, ja ob er überhaupt davon wusste. Jedenfalls hat das Parke-Gutachten Freud zwar ein wenig Geld eingetragen, ihn aber auch in den Geruch gebracht, den früheren Auftraggeber und Partner, die Firma Merck, unfair behandelt zu haben. Welche Anteile die Firma Parke, Davis \& Co. auf dem europäischen Kokain-Markt erobern konnte, wissen wir nicht. Sicher ist, dass sie die führende Stellung der Firma Merck bis zum Ersten Weltkrieg nicht gefährdete.

Nach dem Kriege war das Geschäft mit Kokain in Europa rückläufig. Scriba nennt als Gründe «die Verordnungen gegen den Cocainmissbrauch», «die Einführung ähnlich wirkender synthetisch hergestellter Verbindungen» und «die überall entstehende Concurrenz». ${ }^{50}$ Genaue Zahlen liegen aus dieser Zeit nicht vor. Ganz unbedeutend dürfte die Kokain-Produktion allerdings auch in diesen Jahren angesichts der weiten Verbreitung der Droge nicht gewesen sein - man sprach ja von den «snorting twenties», dem Jahrzehnt der Kokain-Schnupfer, und der Bedarf wurde wohl in erster Linie auf dem deutschen Markt gedeckt, auch wenn seit 1917 Herstellung und Vertriebswege unter die Kontrolle der Opiumverteilungsstelle Berlin gestellt waren. ${ }^{51}$ Ein unbefangener Umgang mit der Droge und ihrer Vermarktung aber schien nicht mehr möglich. Eine gewaltige Kokainwelle hatte inzwischen Europa überschwemmt. Kokain war als Betäubungsmittel eingestuft, der Kokainismus wurde mit einer gefährlichen Infektionskrankheit verglichen, internationale Konferenzen hatten sich mit seiner Bekämpfung befasst, Ärzte, Sozialarbeiter und Polizisten waren überall bemüht, des Drogenmissbrauchs Herr zu werden. ${ }^{52}$ Mit dem zunehmenden Missbrauch hatte auch die Tabuisierung der Droge eingesetzt. Kein Wunder also, dass die Firma Merck wie ihre amerikanischen Konkurrenten nun nicht mehr so offen damit in Verbindung gebracht zu werden suchten. In Festschriften und Darstellungen zur Firmengeschichte wird das lange Zeit so wichtige Kokain kaum je unter den Grosstaten dieser Unternehmen genannt. Dieses Verschweigen ist vielleicht ein Stück der Tabuisierung, die wir im Umgang mit Rauschdrogen bis in unsere Tage beobachten können und die eine offene Auseinandersetzung und eine unvoreingenommene Forschung darüber oft erschweren.

50 Scriba 1926, 1.c. Anm. 10, S. 59.

$51 \mathrm{Vgl}$. Schendzielorz, 1.c. Anm. 14.

$52 \mathrm{Vgl}$. Maier, 1.c. Anm. 14. 